\title{
Robustness of statistical antenna selection for collaborated MIMO reception: A feasibility study
}

\author{
Fengning DU ${ }^{1, \text { a) }}$ and Hidekazu MURATA ${ }^{1}$ \\ ${ }^{1}$ Graduate School of Informatics, Kyoto University, \\ Yoshida-hommachi, Sakyo-ku, Kyoto 606-8501, Japan \\ a)dun@dco.cce.i.kyoto-u.ac.jp
}

Abstract: Collaborated multiple-input multiple-output reception is studied in this letter. This system employs multiple mobile stations (MSs) to receive signals from a base station, and then share their received signals among collaborated MSs. One of important research topic for this system is MS selection for collaboration. This letter presents the relation between the error rate performance of this system and antenna arrangements of the MS side in actual environments. The results suggest that MSs in collaboration can remain the same in terms of long-term average performance.

Keywords: multi-user MIMO, transmission experiment, interference cancellation, terminal collaboration, collaborative reception, measurement campaign

Classification: Wireless Communication Technologies

\section{References}

[1] M. Dohler, J. Dominguez, and H. Aghvami, "Link capacity analysis for virtual antenna arrays," Proc. IEEE 56th Vehicular Technology Conference, vol. 1, pp. 440-443, Sep. 2002. DOI: 10.1109/VETECF.2002.1040381

[2] H. Murata, "Collaborative interference cancellation for multi-user MIMO communication systems," IEICE Technical Report, RCS2013-201, pp. 159-164, Nov. 2013 (in Japanese).

[3] A. Maaref, J. Ma, M. Salem, H. Baligh, and K. Zarin, "Device-centric radio access virtualization for 5G networks," Proc. GLOBECOM 2014 Workshop, Austin, Texas, pp. 887-893, Dec. 2014. DOI: 10.1109/GLOCOMW.2014. 7063545

[4] Y. Kishiyama, "Initial thought on Beyond 5G radio access technologies," IEICE Society Conference, BS-2-2, p. S-11, Sep. 2017.

[5] H. Murata and R. Shinohara, "Performance improvement of ZF-precoded MUMIMO transmission by collaborative interference cancellation," IEICE Commun. Express, vol. 4, no. 5, pp. 155-160, May 2015. DOI: 10.1587/comex.4.155

[6] F. Du and H. Murata, "Performance study of terminal collaborated MIMO reception experimental testbed in actual environment," Proc. 16th IEEE Asia Pacific Wireless Communications Symposium (APWCS2019), Singapore, Aug. 2019. DOI: 10.1109/VTS-APWCS.2019.8851640

[7] H. Murata, R. Shinohara, and Y. Fujimoto, "Performance of adaptive mobile terminal selection schemes for collaborative MMSE linear MIMO detec- 
tion,” IEICE Commun. Express, vol. 8, no. 12, pp. 662-667, Dec. 2019. DOI: 10.1587/comex.2019XBL0122

[8] M. Arai and H. Murata, "Study of adaptive terminal selection schemes for terminal collaborated MIMO reception and its performance evaluation with actual received signals," IEICE Trans. Commun., vol. J103-B, no. 2, pp. 110 118, Feb. 2020. DOI: 10.14923/transcomj.2019GTP0014

[9] T. Koike, H. Murata, and S. Yoshida, "Frequency-domain SC/MMSE iterative equalizer with MF approximation in LDPC-coded MIMO transmissions," IEEE 15th International Symposium on Personal, Indoor and Mobile Radio Communications, vol. 24, pp. 1414-1428, Sep. 2004. DOI: 10.1109/PIMRC.2014. 1373931

[10] S. Minami, H. Nagano, and H. Murata, "Performance evaluation of iterative frequency domain equalization with LDPC code for user collaborated reception,” IEICE Technical Report, RCS2015-383, pp. 291-296, March 2016 (in Japanese).

\section{Introduction}

A multiple-input multiple-output (MIMO) system is a form of transmission scheme for increasing the capacity of a radio link using multiple transmit and receive antennas. Recently, collaborated MIMO reception becomes a new research topic [1, 2, 3, 4]. This system employs multiple mobile stations (MSs) to receive signals from a base station (BS), and then share their received signals among collaborated MSs.

Conventional multiple user MIMO (MU-MIMO) needs instantaneous channel state information (CSI) feedback. It is not suitable for fast fading channels because the information of the channel matrix from the BS to the MSs will change as the movement. The collaborated MIMO reception does not need instantaneous CSI feedback. Therefore, it is suitable for moving MSs [5]. Especially, in public transportation such as bus and train, the reception MSs are close to each other and there is almost no change of the relative position during the movement.

In collaborated MIMO reception systems, the more collaboration MSs we have, the better reception performance can be expected [6]. However, it is better to choose a small subset of collaboration MSs in order to reduce both power consumption and traffic overhead for inter-MS collaboration [7, 8]. If an MS selection scheme fully utilizes instantaneous CSI along with the full knowledge of transmission performance, this MS selection scheme has the best performance and, at the same time, requires heavy overhead traffic for collaboration.

In order to mitigate this overhead traffic problem, a sub-optimum MS selection scheme is required, which utilizes not instantaneous CSI but statistical CSI. In statistical MS selection, the selected MSs offer good average performance for a given period. This should not be affected by realizations of small-scale fading. A question arises: Do the best selected MSs have reproducibility in actual channels? This is exactly the focus of this letter. If the answer is yes, then we can relax the expeditiousness of control signaling for MS selection.

In this letter, we study the relation between the bit error ratio (BER) performance and receive antenna arrangements to answer the question mentioned above. Therefore, we make extensive use of received signal waveforms recorded in actual 
environments. Please note that a statistical MS selection scheme itself is out of the scope of this letter.

\section{System model and signaling}

The system model of collaborated MIMO reception is shown in Fig. 1 (a). BS transmits spatially multiplexed signal streams by using multiple antennas. The MSs receive these signals and transmit them to other MSs by short-range highspeed wireless communications. Thus, the signals received by MSs are utilized for demodulation in collaborative manner.

In this letter, as shown in Fig. 1 (b), packets are transmitted in every $50 \mathrm{~ms}$ frame by using quadrature phase-shift keying (QPSK) modulation (312.5 kilo symbols per second). The packet consists of a synchronization sequence (SW), one of orthogonal training sequences (TS), a control sequence (CTRL), a cyclic prefix (CP), a data section (DATA), and a cyclic redundancy check (CRC).

Frequency-domain soft-cancel/minimum mean square error (MMSE) iterative equalizer is employed. The received signals are equalized and separated by MMSE filters in frequency domain. The soft values are calculated by belief propagation for low density parity check code $[9,10]$.

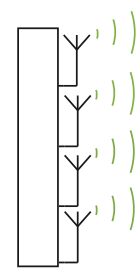

BS

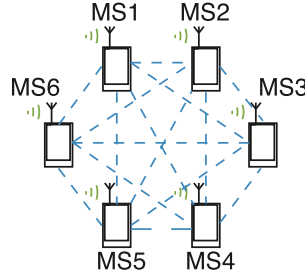

(a)

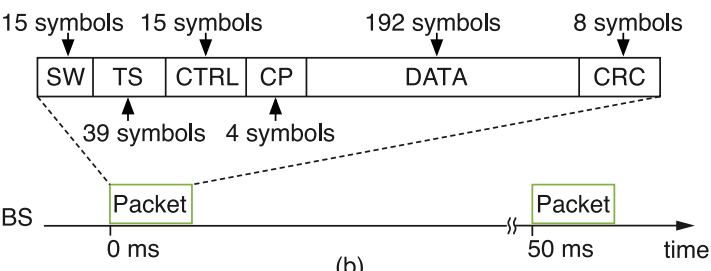

(b)

Fig. 1. System model and signaling format. (a) System model. (b) Signaling format.

\section{Experimental setup}

As shown in Fig. 2 (a), there were four BS antennas on the roof of the building. The antenna height was $25.5 \mathrm{~m}$ above the ground. The carrier frequency was $427.2 \mathrm{MHz}$. The transmit power per antenna was $1 \mathrm{~W}$. As shown in Fig. 2 (b), six MSs' receive antennas were set on the roof of a vehicle ( $2.1 \mathrm{~m}$ height). Two arrangements of MS antennas, namely a uniform circular array (UCA) arrangement and a trapezoid arrangement, were employed. The antenna gains of BS and MS antennas were $5.8 \mathrm{dBi}$ and $2.15 \mathrm{dBi}$, respectively. The timings and frequencies of the entire system 


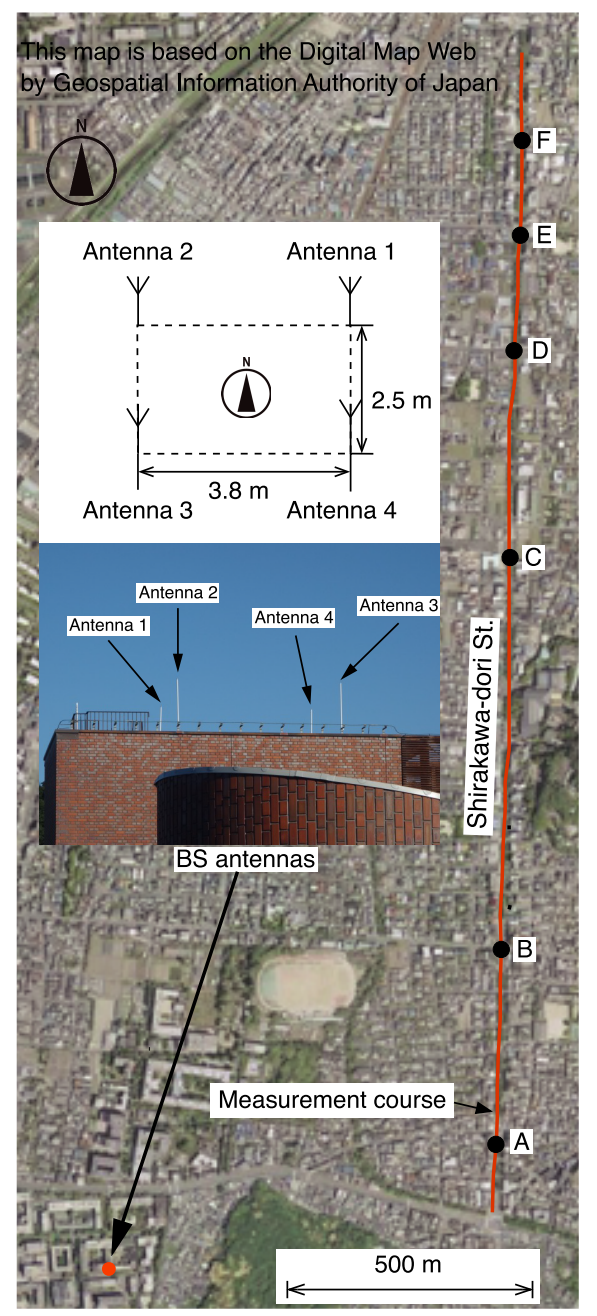

(a)
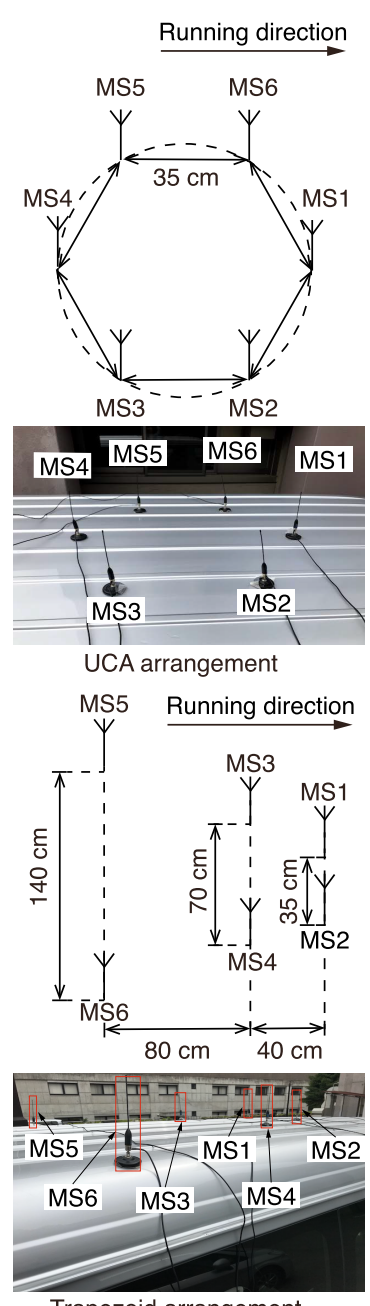

Trapezoid arrangement

(b)

Fig. 2. Measurement campaign scenario. (a) Measurement course. (b) Arrangements of MS antennas.

were based on 1-pulse-per-second signals and $10 \mathrm{MHz}$ signals of global positioning system receivers.

In this letter, a subset of the six received signals from BS were selected and used for equalization/demodulation. In order to examine the BERs of all possible signal combinations, the received signal waveforms from BS were recorded at each MS and used for offline processing. Therefore, there was no inter-MS communication for collaboration in this letter. As shown in Fig. 2 (a), we drove a vehicle on Shirakawadori street in Kyoto city to record the actual received signal waveforms. The received signals while the vehicle stopped at traffic lights (A, B, C, D, E, F in Fig. 2 (a)) were not used in offline processing.

By examining all possible combinations of six signals, we compared the average BER performance of all signal combinations. Please note that the combination remained fixed during the entire measurement course. Received power calibration was performed for all MSs before the experiment. 


\section{Experimental results}

Figs. 3 (a) and (b) show the empirical cumulative distribution function (CDF) of BER averaged over four streams in a frame. We conducted the experiments twice (trial 1 and 2) for both UCA and trapezoid arrangements. From these figures, it is clearly confirmed that the BER performance is improved as the number of signals for equalization/demodulation is increased. At the top of each graph, the average received power of each MS is shown. In these figures, each CDF curve corresponds to a specific signal combination.

In Fig. 3 (a), the same signal combinations perform well in both trials. However, there are relatively small variations in the $\mathrm{CDF}$ curves of signal combinations for the UCA arrangement. On the other hand, we can see larger variations in CDF curves
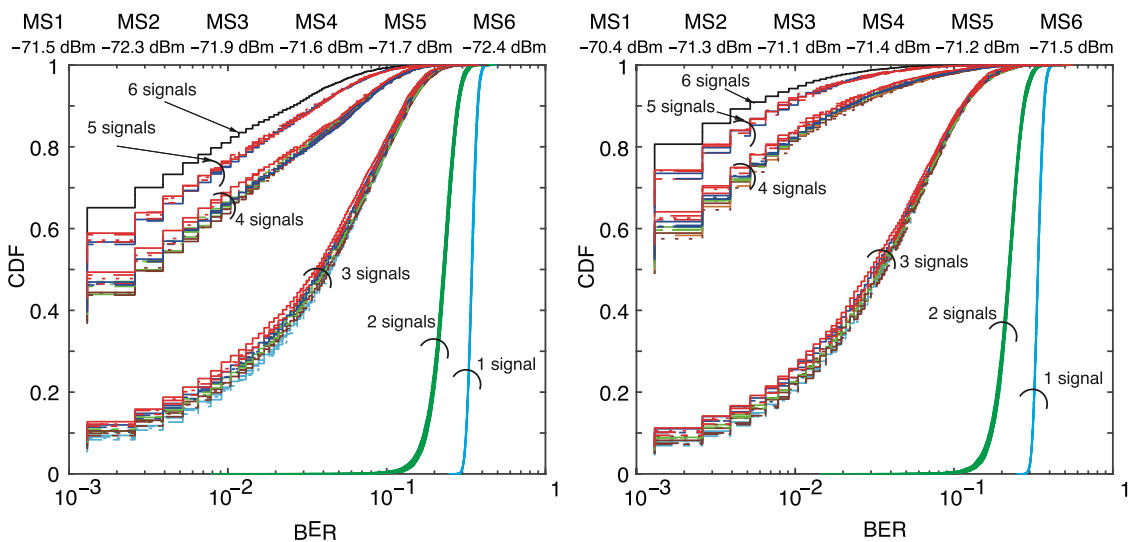

(a) CDF of BER performance in UCA arrangement (left: trial 1, right: trial 2).
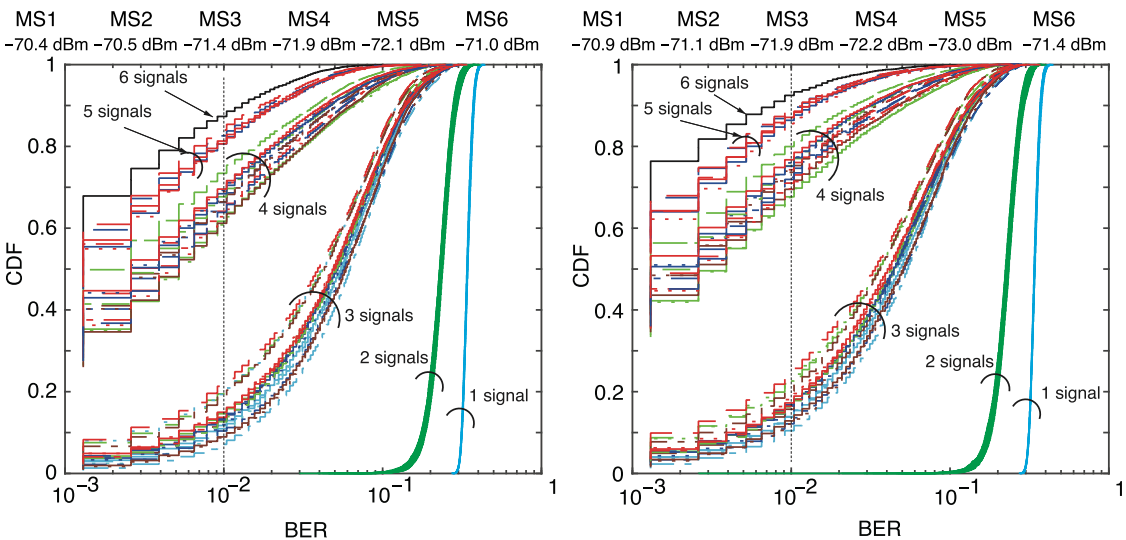

(b) CDF of BER performance in trapezoid arrangement (left: trial 1, right: trial 2).

\begin{tabular}{|c|c|c|c|c|c|c|c|c|c|c|c|c|c|c|c|c|c|c|c|c|}
\hline \multirow{2}{*}{$\frac{\text { CDF in (b) }}{3 \text { signals, trial } 1}$} & \multicolumn{3}{|c|}{ large CDF } & \multicolumn{15}{|c|}{ Signal combinations in the order of descending CDF at BER $=10^{-2}$} & \multicolumn{2}{|c|}{ small CDF } \\
\hline & 456 & 145 & 146 & 156 & 346 & 345 & 134 & 356 & 246 & 245 & 136 & 256 & 135 & 124 & 125 & 234 & 126 & 236 & 235 & 123 \\
\hline 3 signals, trial 2 & 456 & 146 & 145 & 156 & 346 & 345 & 246 & 134 & 245 & 356 & 256 & 136 & 135 & 125 & 126 & 124 & 236 & 234 & 235 & 123 \\
\hline 4 signals, trial 1 & 1456 & 3456 & 1346 & 1345 & 2456 & 1356 & 1256 & 1246 & 1245 & 2346 & 2356 & 2345 & 1234 & 1236 & 1235 & & & & & \\
\hline 4 signals, trial 2 & 1456 & 3456 & 1346 & 1356 & 2456 & 1345 & 1256 & 1246 & 1245 & 2356 & 2346 & 2345 & 1236 & 1234 & 1235 & & & & & \\
\hline 5 signals, trial 1 & 13456 & 12456 & 23456 & 12345 & 12356 & 12346 & & & & & & & & & & & & & & \\
\hline 5 signals, trial 2 & 13456 & 12456 & 23456 & 12356 & 12346 & 12345 & & & & & & & & & & & & & & \\
\hline
\end{tabular}

(c) Signal combinations in the order of descending CDF.

Fig. 3. Empirical CDF of frame by frame BER and order of CDF values. 
for the trapezoid arrangement shown in Fig. 3 (b). Please note that absolute values of CDF are inevitably different between trial 1 and trial 2 due to different traffic condition (vehicle speed, lane position, other vehicles).

To investigate the relation between the CDF performance and a signal combination for the trapezoid arrangement in more detail, Fig. 3 (c) shows the signal combinations in the order of descending $\mathrm{CDF}$ values at $\mathrm{BER}=10^{-2}$. The digit string shown in Fig. 3 (c) indicate a signal combination. For example, 'xyz' corresponds to the signals received at MS $x, \mathrm{MS} y, \mathrm{MS} z$ which has been selected and used for offline processing.

As can be seen, the signal combinations that have the largest CDF values at $\mathrm{BER}=10^{-2}$ are consistent in both trials. In the three signals selection case, the signal combinations of '145', '146', '156' and '456' have better BER performance (largest CDF value) than others in both trials. Also in the four signals selection case, the signal combination of ' 1456 ' has achieved the best performance in both trials. The same can be observed for the five signals selection case.

\section{Conclusion}

This letter has presented the measurement results of the BER performance of the collaborated MIMO reception system. The performance of this system in actual environments was studied. In our experiment, when the BS transmits four signal streams, the BER performance has been examined up to six MSs.

The experimental results show that the best signal combinations are consistent in both trials, although the measurement environments are slightly different. This difference inevitably involves different small-scale fading. This result suggests that a statistical MS selection scheme can have robustness in actual environments.

\section{Acknowledgments}

This work was supported by the JSPS KAKENHI Grant Number JP15H04007 and SCOPE of the Ministry of Internal Affairs and Communications. 